\title{
Nonlinear Transformations for Accelerating the Convergence of $M$-Dimensional Series
}

\author{
By N. C. Albertsen, G. Jacobsen and S. B. Sørensen
}

\begin{abstract}
Two transformations for accelerating the convergence of infinite $M$-dimensional series are presented. They are generalizations of Shanks' $e$-transformation and Levin's one-dimensional transformations, respectively.

The transformations are compared with an $M$-dimensional transformation by Levin in numerical examples.
\end{abstract}

1. Introduction. There exists a large number of methods for accelerating convergence of series and sequences. Some of the most important ones are the $e$-transformation of Shanks [8], the $\theta$-algorithm of Brezinski [2] and the transformations of Levin [7]. These methods are all transformations of one-dimensional series, but extensions by Streit [9], Chisholm [3], and Levin [6] deal with two-dimensional and $M$-dimensional series.

We will here present two new transformations for accelerating convergence of $M$-dimensional series. The first is an extension of Shanks' $e$-transformation and the second of Levin's one-dimensional transformations.

A short review of Streit's, Chisholm's and Levin's two-dimensional transformations is given in Section 2, and the new transformations are presented in Sections 3 and 4. Sections 5 and 6 contain numerical examples where the new methods are compared with Levin's transformation.

2. A Review of Streit's, Chisholm's and Levin's 2-Dimensional Transformations. The $e$-transformation by Shanks [8] is generalized by Streit [9] to two dimensions, but the generalization only includes the first order $e$-transformation. Generalizations of the higher order $e$-transformations are given by Levin [6] and generalizations of Padé approximants [1] by Chisholm [3].

Chisholm defines in [3] rational approximations to a function of two variables given by a power series

$$
f(x, y)=\sum_{i=0}^{\infty} \sum_{j=0}^{\infty} c_{i j} x^{i} y^{j} .
$$

The approximants have the form

$$
f_{M / M}=\frac{\sum_{i=0}^{M} \sum_{j=0}^{M} a_{i j} x^{i} y^{j}}{\sum_{k=0}^{M} \sum_{l=0}^{M} b_{k l} x^{k} y^{l}}
$$

Received August 9, 1982; revised January 27, 1983.

1980 Mathematics Subject Classification. Primary 65B10. 
and are defined very similarly to the one-dimensional Padé approximants. The Chisholm approximants can be considered as a two-dimensional generalization of the Padé approximants. A further generalization to $M$-dimensions is given by Chisholm and McEwan [4].

Two-dimensional approximants with different maximum powers in numerator and denominator are defined by Graves-Morris, Hughes-Jones and Makinson [5].

Another transformation of $M$-dimensional series is given by Levin [6]. It has the property that it sums series exactly whose terms satisfy a linear difference equation.

The Shanks $e$-transformation [8] has the same property for one-dimensional series, and Levin's transformation is in that sense a generalization of Shanks' transformation. Levin's approximation for the sum of a two-dimensional series,

$$
A=\sum_{i=0}^{\infty} \sum_{j=0}^{\infty} a_{i j}
$$

is calculated by solving the system of equations

$$
A_{s t}=\Omega+\sum_{(t, j) \in R} \beta_{i j} a_{s+t, t+j}, \quad(s, t) \in S,
$$

where $\Omega$ is the approximation to the sum, and

$$
A_{s t}=\sum_{(i, j) \in B} a_{i j}, \quad B=\{(i, j) \mid 0 \leqslant i \leqslant s \text { or } 0 \leqslant j \leqslant t\} .
$$

The transformation is in Levin's notation denoted by

$$
\Omega=[n ; m / k]_{r},
$$

where $m$ and $k$ are parameters that define the sets $R$ and $S$.

$$
\begin{aligned}
& S=\{(s, t) \mid m-k \leqslant s \leqslant m \text { and } m-k \leqslant t \leqslant m\}, \\
& R=\{(i, j) \mid r-m \leqslant i \leqslant r+k-m \\
& \quad \text { and } r-m \leqslant j \leqslant r+k-m\} \backslash\{(r+t-m, r+t-m)\} .
\end{aligned}
$$

It is seen that calculation of (2.5) involves infinite one-dimensional sums, which are determined by ordinary Padé approximants. The first parameter $n$ in (2.6) is the order of the $[n / n]$ Padé approximant used.

3. A Generalization of Shanks' Transformation. A necessary and sufficient condition that Shanks' $e$-transformation [8] gives the exact limit of a sequence $A_{n}$, is that $A_{n}$ satisfy a linear difference equation of the form

$$
\gamma_{0}\left(A_{n}-A\right)+\cdots+\gamma_{k}\left(A_{n+k}-A\right)=0, \quad \gamma_{0} \gamma_{k} \neq 0, \sum_{i=0}^{k} \gamma_{k} \neq 0,
$$

where $A$ is the limit of $A_{n}$ and $\gamma_{0}, \ldots, \gamma_{k}$ are constants.

The transformation has the property of eliminating exponential transients from the sequence, which follows from the fact that any sequence of the form

$$
A_{n}=A+\sum_{1}^{k} c_{i} q_{i}^{n} \quad\left(q_{i} \neq 0,1\right)
$$

satisfies (3.1). The $e$-transformation can therefore determine the exact limit $A$ of (3.2) from a finite number of values $A_{n}$ for both convergent $\left(\left|q_{i}\right|<1\right)$ and divergent $\left(\left|q_{i}\right|>1\right)$ sequences. 
In the following we will generalize the equation (3.1) to $M$-dimensions and define a transformation that determines the exact limit of sequences that satisfies the generalized form of (3.1). We have considered it instructive first to generalize (3.2) to show that the $M$ th-order transformation also has the property of eliminating exponential transients. The $M$ th order equivalent to (3.2) is the sequence

$$
\begin{gathered}
A_{n_{1}, n_{2}, \ldots, n_{\mathrm{M}}}=A+\sum_{t=1}^{k} c_{t} q_{t, 1}^{n_{1}} q_{t, 2}^{n_{2}} \cdots q_{t, M}^{n_{M},}, \\
q_{t, 1} q_{t, 2}, \ldots, q_{t, M} \neq 0,\left(q_{t, 1} q_{t, 2}, \ldots, q_{t, M}\right) \neq(1,1, \ldots 1) .
\end{gathered}
$$

In order to simplify the derivation we will first consider the 2-dimensional case in detail;

$$
A_{n m}=A+\sum_{t=1}^{k} c_{t} q_{t}^{n} p_{t}^{m}, \quad q_{t}, p_{t} \neq 0,\left(q_{t}, p_{t}\right) \neq(1,1),
$$

and the generalization to $M$ dimensions will be explained at the end of this section.

The transformation can also accelerate the convergence of a series if it is used on a sequence $A_{n m}$ defined by

$$
A_{n m}=\sum_{i=0}^{n} \sum_{j=0}^{m} a_{i j}
$$

or

$$
A_{n m}=\sum_{(i, j) \in B} a_{i j}, \quad B=\{(i, j) \mid 0 \leqslant i \leqslant n \text { or } 0 \leqslant j \leqslant m\} .
$$

In the derivation we shall use a sequence $R_{n m}$ which is equal to (3.4) without the constant term $A$ :

$$
R_{n m}=A_{n m}-A,
$$

and

$$
R_{n m}=\sum_{t=1}^{k} c_{t} q_{t}^{n} p_{t}^{m}
$$

We will now prove that a set of numbers $\gamma_{i j}$ (not all equal to zero) exists, such that the linear difference equation

$$
\sum_{(i, j) \in D} \gamma_{i j} R_{n+i, m+j}=0
$$

is satisfied for all integer values of $n$ and $m$. The set $D$ contains $k+1$ ordered pairs of integer numbers $(i, j)$, which can be chosen arbitrarily. If we substitute (3.8) into (3.9), we have

$$
\sum_{t=1}^{k} c_{t} q_{t}^{n} p_{t}^{m} \sum_{(i, j)(l)} \gamma_{1}, q_{t}^{\prime} p_{t}^{\prime} \quad() .
$$

and this shows that (3.9) is satisfied by choosing the $\gamma_{1}$, 's as a nontrivial solution to the set of linear equations

$$
\sum_{(i, j) \in D} \gamma_{i j} q_{t}^{i} p_{t}^{j}=0, \quad t=1,2, \ldots, k
$$


Nontrivial solutions to (3.11) always exist because there are $k$ equations, but $k+1$ unknowns $\gamma_{i j}$.

We introduce an ordering of the elements in $D$ by defining two functions $\omega_{1}(j)$ and $\omega_{2}(j)$, so that

$$
\left(\omega_{1}(j), \omega_{2}(j)\right), \quad j=1, \ldots, k+1,
$$

generates the $k+1$ elements in $D$. Equation (3.9) can now be written

$$
\sum_{j=1}^{k+1} \gamma_{\omega_{1}(j), \omega_{2}(j)} R_{n+\omega_{1}(j), m+\omega_{2}(j)}=0
$$

or as

$$
\sum_{j=1}^{k+1} \gamma_{\omega_{1}(j), \omega_{2}(j)}\left(A_{n+\omega_{1}(1), m+\omega_{2}(j)}-A\right)=0
$$

by inserting (3.7). We define another set $E$ of pairs of integer numbers. The set contains $k+1$ elements, which are ordered by the functions $\phi_{1}(i)$ and $\phi_{2}(i)$, so that

$$
\left(\phi_{1}(i), \phi_{2}(i)\right), \quad i=1,2, \ldots, k+1,
$$

generates the elements in E. Equation (3.14) is valid for all integer values of $n$ and $m$, and we therefore have

$$
\sum_{j=1}^{k+1} \gamma_{\omega_{1}(j), \omega_{2}(j)}\left(A_{\phi_{1}(1)+\omega_{1}(j), \phi_{2}(l)+\omega_{2}(j)}-A\right)=0 .
$$

If we use the notation

$$
V_{i j}=A_{\phi_{1}(i)+\omega_{1}(1), \phi_{2}(1)+\omega_{2}(1)}, \quad \Delta V_{1 j}=V_{i+1, j}-V_{i j},
$$

the rule of Cramer shows that

$$
\left|\begin{array}{ccc}
\left(V_{11}-A\right) & \cdots & \left(V_{1, k+1}-A\right) \\
\vdots & & \vdots \\
\left(V_{k+1,1}-A\right) & \cdots & \left(V_{k+1, k+1}-A\right)
\end{array}\right|=0,
$$

since not all $\gamma$ 's in (3.16) are equal to zero. From (3.18) we can find the constant $A$. By subtraction of row number $k$ from row number $k+1$, number $k-1$ from number $k$ etc., we obtain

$$
\left|\begin{array}{ccc}
\left(V_{11}-A\right) & \cdots & \left(V_{1, k+1}-A\right) \\
\Delta V_{11} & \cdots & \Delta V_{1, k+1} \\
\vdots & & \vdots \\
\Delta V_{k, 1} & \cdots & \Delta V_{k, k+1}
\end{array}\right|=0
$$

and therefore

$$
A=\left|\begin{array}{ccc}
V_{11} & \cdots & V_{1, k+1} \\
\Delta V_{11} & \cdots & \Delta V_{1, k+1} \\
\vdots & \cdots & \vdots \\
\Delta V_{k, 1} & \cdots & \Delta V_{k, k+1}
\end{array}\right| /\left|\begin{array}{ccc}
1 & \cdots & 1 \\
\Delta V_{11} & \cdots & \Delta V_{1, k+1} \\
\vdots & \cdots & \vdots \\
\Delta V_{k, 1} & \cdots & \Delta V_{k, k+1}
\end{array}\right|,
$$

assuming that the determinant in the denominator is nonsingular. 
We denote the transformation defined by (3.20) of a sequence $A_{n m}$ by $S\left(A_{n m}\right)$. It is easily seen that the transformation is nonlinear, but it follows from the definition (3.20) that $S$ is homogeneous and translative:

$$
\begin{aligned}
S\left(\alpha A_{n m}\right) & =\alpha S\left(A_{n m}\right), \\
S\left(A_{n m}+C\right) & =S\left(A_{n m}\right)+C,
\end{aligned}
$$

where $C$ is a constant sequence.

In the numerical examples of Sections 5 and 6 we use a notation similar to that of Levin's two-dimensional transformation. The sets $D$ and $E$ are defined as

$$
\begin{aligned}
& D=\{(i, j) \mid m-k \leqslant i \leqslant m \text { and } m-k \leqslant j \leqslant m\}, \\
& E=\{(i, j) \mid 0 \leqslant i \leqslant k \text { and } 0 \leqslant j \leqslant k\},
\end{aligned}
$$

and the transformation is denoted

$$
A=S[m, k]
$$

when the partial sums (3.5) are used, and denoted

$$
A=S^{\prime}[n ; m, k]
$$

when (3.6) is used. The calculation of (3.6) involves infinite one-dimensional sums which are determined by use of ordinary Padé approximants. The parameter $n$ in (3.25) is the order of the $[n / n]$ Padé approximant used.

The transformation is easily generalized to $M$-dimensions. The approximation to the sum of an $M$-dimensional sum is still given by (3.20), if the partial sums (3.5) and (3.6) are replaced by

$$
A_{s_{1}, s_{2} \ldots s_{M}}=\sum_{i_{1}=0}^{s_{1}} \ldots \sum_{i_{M}=0}^{s_{M}} a_{i_{1}, i_{2} \ldots \ldots i_{M}}
$$

or

$$
\begin{gathered}
A_{s_{1}, s_{2} \ldots s_{M}}=\sum_{\left(i_{1}, \ldots, i_{M}\right) \in B} a_{i_{1}, i_{2} \ldots i_{M}}, \\
B=\left\{\left(i_{1}, \ldots, i_{M}\right) \mid 0 \leqslant i_{1} \leqslant s_{1} \text { or } \cdots \text { or } 0 \leqslant i_{M} \leqslant s_{M}\right\},
\end{gathered}
$$

and the sets $D$ and $E$ are replaced by

$$
\begin{aligned}
& D=\left\{\left(i_{1}, \ldots, i_{M}\right) \mid m-k \leqslant i_{1} \leqslant m \text { and } \cdots \text { and } m-k \leqslant i_{M} \leqslant m\right\}, \\
& E=\left\{\left(i_{1}, \ldots, i_{M}\right) \mid 0 \leqslant i_{1} \leqslant k \text { and } \cdots \text { and } 0 \leqslant i_{M} \leqslant k\right\} .
\end{aligned}
$$

It is seen that calculation of (3.27) involves infinite $(M-1)$-dimensional sums.

4. A Generalization of Levin's One-Dimensional Transformations. We start with a derivation of the one-dimensional transformations of Levin [7]. The derivation is different from that in [7] and is included because it explains how the transformations work and therefore facilitates the generalization to $M$ dimensions.

For the one-dimensional series

$$
\sum_{i=0}^{\infty} a_{i}
$$

with the sum $A$, we define the partial sum $A_{n}$ by

$$
A_{n}=\sum_{i=0}^{n} a_{i}
$$


and therefore

$$
A-A_{n}=\sum_{i=n \rightarrow 1}^{\infty} a_{i}
$$

It is often possible to find a sequence $R_{n}$ which gives the leading behavior of (4.3). That means ( $c$ is a constant)

$$
\frac{A-A_{n}}{R_{n}} \rightarrow c \text { for } n \rightarrow \infty
$$

The behavior of the left-hand side of (4.4) as a function of $n$ is often simpler than (4.3), because the leading factor is removed. Thus, it is easier to approximate (4.4) than (4.3) by a series of simple functions. We choose

$$
\frac{A-A_{n}}{R_{n}} \cong c_{0}+\frac{c_{1}}{(n+1)}+\frac{c_{2}}{(n+1)^{2}}+\cdots+\frac{c_{k}}{(n+1)^{k}},
$$

which is equivalent to

$$
A_{n} \cong A-R_{n}\left(c_{0}+\frac{c_{1}}{(n+1)}+\frac{c_{2}}{(n+1)^{2}}+\cdots+\frac{c_{k}}{(n+1)^{k}}\right) .
$$

By inserting values of $A_{n}$ and $R_{n}$ into (4.6) we obtain a system of equations which can be solved for the constant $A$ giving an approximate value of the sum (4.1).

This is the general form of the one-dimensional transformations suggested by Levin in [7], where also three suggestions for the choice of $R_{n}$ are given, leading to three transformations

$$
\begin{array}{ll}
R_{n}=a_{n} & (t \text { transformation }), \\
R_{n}=n a_{n} & (u \text { transformation }), \\
R_{n}=\frac{a_{n} a_{n+1}}{a_{n+1}-a_{n}} & (v \text { transformation }) .
\end{array}
$$

The transformation (4.6) is easily generalized to two dimensions. We consider the double series

$$
\sum_{i=0}^{\infty} \sum_{j=0}^{\infty} a_{i j}
$$

with the $\operatorname{sum} A$, and define the partial sum $A_{s t}$ by

$$
A_{s t}=\sum_{(i, j) \in B} a_{i j}, \quad B=\{(i, j) \mid 0 \leqslant i \leqslant s \text { or } 0 \leqslant j \leqslant t\} .
$$

In analogy with (4.3), (4.4) and (4.6) we have

$$
A-A_{s t}=\sum_{i=s+1}^{\infty} \sum_{j=t+1}^{\infty} a_{i j}
$$

and

$$
\frac{A-A_{s t}}{R_{s t}} \rightarrow c \text { for } s \rightarrow \infty \text { and } t \rightarrow \infty
$$


Here $R_{s t}$ is a sequence which gives the leading behavior of (4.10). The general two-dimensional transformation has the form

$$
A_{s t} \cong A-R_{s t} \sum_{(i, j) \in D} \frac{c_{i j}}{(s+1)^{i}(t+1)^{j}} .
$$

In the numerical examples we have chosen

$$
R_{s t}=a_{s+1, t+1},
$$

and the infinite one-dimensional sums in (4.9) are approximated by $[n / n]$ Padé approximants. We denote the transformation

$$
A=L[n ; m, k],
$$

and it is calculated by solving the systems of equations

$$
\begin{aligned}
A_{s t}=A-a_{s+1, t+1} \sum_{(i, j) \in D} \frac{c_{i j}}{(s-m+k+1)^{i}(t-m+k+1)^{j}}, & \\
& (s, t) \in E,
\end{aligned}
$$

where

$$
\begin{aligned}
& D=\{(i, j) \mid 0 \leqslant i \leqslant k \text { and } 0 \leqslant j \leqslant k\} \backslash\{(k, k)\}, \\
& E=\{(s, t) \mid m-k \leqslant s \leqslant m \text { and } m-k \leqslant t \leqslant m\} .
\end{aligned}
$$

It follows directly from the definition (4.15) that the transformation is nonlinear, but homogeneous and translative as defined by (3.21) and (3.22).

The generalization of (4.12) to $M$-dimensions is straightforward:

$$
A_{s_{1} \ldots s_{M}} \cong A-R_{s_{1} \ldots s_{M}} \cdot \sum_{\left(i_{1} \ldots i_{M}\right) \in D} \frac{c_{i_{1} \ldots i_{M}}}{\left(s_{1}+1\right)^{i_{1}} \cdots\left(s_{M}+1\right)^{i_{M}}},
$$

where

$$
\begin{gathered}
A_{s_{1} \ldots s_{Y}}=\sum_{\left(i_{1} \ldots i_{M}\right) \in B} a_{i_{1} \ldots i_{M},}, \\
B=\left\{\left(i_{1}, \ldots, i_{M}\right) \mid 0 \leqslant i_{1} \leqslant s_{1} \text { or } \cdots \text { or } 0 \leqslant i_{M} \leqslant s_{M}\right\},
\end{gathered}
$$

and the function $R$ can be chosen

$$
R_{s_{1} \ldots s_{M}}=a_{s_{1}+1 \ldots s_{M}+1} .
$$

The evaluation of (4.18) involves infinite $(M-1)$-dimensional sums.

5. The Beta Function. Numerical Example. In this section we apply the approximants of Sections 3 and 4 and the $[n ; m / k]$ transformation of Levin to a double power series

$$
f(x, y)=\sum_{i=0}^{\infty} \sum_{j=0}^{\infty} c_{i j} x^{i} y^{j}
$$

which is defined by the Beta function

$$
\begin{aligned}
& B(1+x, 1+y)=\frac{1+x y f(x, y)}{(1+x)(1+y)}, \\
& B(1+x, 1+y)=\frac{\Gamma(1+x) \Gamma(1+y)}{\Gamma(2+x+y)} .
\end{aligned}
$$


The example was originally considered by Graves-Morris, Hughes-Jones and Makinson [5], who used the generalized form of Chisholm's approximants mentioned in Section 2. Levin [6] has considered the same example using his two-dimensional generalization (2.4) of Shanks' $e$-transformation [8].

TABLE 5.1

Comparison between $[n ; m, k]_{r}, S^{\prime}[n ; m, k]$ and $L[n ; m, k]$ approximants to $B(p, q)$, with solution of the same number of linear equations.

\begin{tabular}{|l|c|c|c|c|c|} 
& Terms & Equations & & & \\
\hline $\mathrm{p}, \mathrm{q}$ & & & $1.75,-0.25$ & $1.75,-0.5$ & $1.75,-0.75$ \\
\hline $\mathrm{B}(\mathrm{p}, \mathrm{q})$ & & & -5.08327850876 & -3.59442070421 & -4.44288293816 \\
\hline$[10 ; 3 / 3]_{4}$ & 167 & 16 & -5.0832785077 & -3.594420696 & -4.44288285 \\
$\mathrm{~S}^{\prime}[10 ; 3,3]$ & 245 & 16 & -5.08327850874 & -3.59442070416 & -4.44288293803 \\
$\mathrm{~L}[10 ; 3,3]$ & 153 & 16 & -5.0832784908 & -3.59442067 & -4.44288284 \\
\hline
\end{tabular}

\begin{tabular}{|l|c|l|l|l|l|} 
& Terms & Equations & & & \\
\hline $\mathrm{p}, \mathrm{q}$ & & & $1.75,-1.25$ & $1.75,-1.5$ & $1.75,-1.75$ \\
\hline $\mathrm{B}(\mathrm{p}, \mathrm{q})$ & & & 2.0333114035 & 0.599070117 & 0 \\
\hline$[10 ; 3 / 3] 4$ & 167 & 16 & 2.0333089 & 0.599063 & -0.00004 \\
$\mathrm{~S}^{\prime}[10 ; 3,3]$ & 245 & 16 & 2.033311405 & 0.599070119, & 0.00000002 \\
$\mathrm{~L}[10 ; 3,3]$ & 153 & 16 & 2.0333091 & 0.599062 & -0.00005 \\
\hline
\end{tabular}

\begin{tabular}{|l|c|c|l|l|l|} 
& Terms & Equations & & & \\
\hline $\mathrm{p}, \mathrm{q}$ & & & $-0.25,-0.25$ & $-0.5,-0.5$ & $-0.75,-0.75$ \\
\hline $\mathrm{B}(\mathrm{p}, \mathrm{q})$ & & & -6.777704678 & 0 & 9.8883983 \\
\hline$[10 ; 3 / 3] 4$ & 167 & 16 & -6.77770450 & -0.0000023 & 9.88844 \\
$\mathrm{~S}^{\prime}[10 ; 3,3]$ & 245 & 16 & -6.77770471 & -0.00000022 & 9.8883964 \\
$\mathrm{~L}[10 ; 3,3]$ & 153 & 16 & -6.77770715 & -0.0000042 & 9.88841 \\
\hline
\end{tabular}

\begin{tabular}{|l|l|l|l|l|l|} 
& Terms & Equations & & & \\
\hline $\mathrm{p}, \mathrm{q}$ & & & $-1.25,-1.25$ & $-1,5,-1.5$ & $-1.75,-1.75$ \\
\hline $\mathrm{B}(\mathrm{p}, \mathrm{q})$ & & & -16.26649 & 0 & $? 8.253$ \\
\hline$[10 ; 3 / 3] 4$ & 167 & 16 & -16.270 & -0.017 & 28.11 \\
$\mathrm{~S}^{\prime}[10 ; 3,3]$ & 245 & 16 & -16.26659 & -0.00063 & 28.248 \\
$\mathrm{~L}[10 ; 3,3]$ & 153 & 16 & -16.269 & -0.016 & 28.12 \\
\hline
\end{tabular}


TABLE 5.2

Comparison between $[n ; m / k]_{r}, S^{\prime}[n ; m, k]$ and $L[n: m, k]$ approximants to $B(p, q)$, with approximately the same series terms used.

\begin{tabular}{|l|c|c|c|c|c|} 
& Terms & Equations & & & \\
\hline$p, q$ & & & $-0.25,-0.25$ & $-0.5,-0.5$ & $-0.75,-0.75$ \\
\hline$B(p, q)$ & & & -6.777704678 & 0 & 9.88839828 \\
\hline$[7 ; 4 / 3]_{3}$ & 128 & 16 & -6.777699 & 0.000032 & 9.8886 \\
$S^{\prime}[7 ; 2,2]$ & 125 & 9 & -6.7779 & 0.00058 & 9.8885 \\
$L[7 ; 4,4]$ & 126 & 25 & -6.777704680 & -0.0000000050 & 9.88839825 \\
\hline
\end{tabular}

\begin{tabular}{|l|c|c|l|l|l|} 
& Terms & Equations & & & \\
\hline $\mathrm{p}, \mathrm{q}$ & & & $-1.25,-1.25$ & $-1.5,-1.5$ & $-1.75,-1.75$ \\
\hline $\mathrm{B}(\mathrm{p}, \mathrm{q})$ & & & -16.26649 & 0 & 28.2526 \\
\hline$[7 ; 4 / 3]_{3}$ & 128 & 16 & -16.258 & -0.057 & 28.7 \\
$\mathrm{~S}^{\prime}[7 ; 2,2]$ & 125 & 9 & -16.32 & -0.21 & 27.5 \\
$\mathrm{~L}[7 ; 4,4]$ & 126 & 25 & -16.26648 & 0.000038 & 28.2533 \\
\hline
\end{tabular}

\begin{tabular}{|l|c|c|c|c|c|} 
& Terms & Equations & & & \\
\hline $\mathrm{p}, \mathrm{q}$ & & & $1.75,-0.25$ & $1.75,-0.5$ & $1.75,-0.75$ \\
\hline $\mathrm{B}(\mathrm{p}, \mathrm{q})$ & & & -5.08327850876 & -3.59442070421 & -4.442882938 \\
\hline$[7 ; 4 / 3]_{3}$ & 128 & 16 & -5.08327854 & -3.59442078 & -4.4428832 \\
$\mathrm{~S}^{\prime}[7 ; 2,2]$ & 125 & 9 & -5.0832782 & -3.5944201 & -4.442882 \\
$\mathrm{~L}[7 ; 4,4]$ & 126 & 25 & -5.08327850880 & -3.59442070423 & -4.442882935 \\
\hline
\end{tabular}

\begin{tabular}{|l|l|l|l|l|l|} 
& Terms & Equations & & & \\
\hline $\mathrm{p}, \mathrm{q}$ & & & $1.75,-1.25$ & $1.75,-1.5$ & $1.75,-1.75$ \\
\hline $\mathrm{B}(\mathrm{p}, \mathrm{q})$ & & & 2.033311 & 0.59907 & 0 \\
\hline$[7 ; 4 / 3]_{3}$ & 128 & 16 & 2.033309 & 0.59906 & 0.000010 \\
$\mathrm{~S}^{\prime}[7 ; 2,2]$ & 125 & 9 & 2.03332 & 0.59910 & 0.00013 \\
$\mathrm{~L}[7 ; 4,4]$ & 126 & 25 & 2.033312 & 0.59908 & 0.000077 \\
\hline
\end{tabular}

We have calculated the coefficients of (5.1) by the first procedure suggested in [5]. Reference results for the Beta function have been calculated using (5.3).

In Table 5.1 we compare transformations which require the same number of linear equations to be solved. Table 5.2 shows a comparison of transformations which use 
approximately the same series terms. Because the transformations are defined differently, it is not possible to compare transformations that use exactly the same series terms.

The number, $I$, of series terms can be evaluated by

$$
\begin{gathered}
I=2(2 n+1)(m+1)-(m+1)^{2}+(k+r-m)^{2}-1, \\
\quad \text { when } k+r-m>0, \\
I=2(2 n+1)(m+1)-(m+1)^{2}, \quad \text { when } k+r-m \leqslant 0,
\end{gathered}
$$

for the $[n ; m / k]_{r}$ approximant,

$$
I=(m+k+1)^{2}
$$

for the $S[m, k]$ transformation,

$$
I=2(2 n+1)(m+k+1)-(m+k+1)^{2}
$$

for the $S^{\prime}[n ; m, k]$ transformation, and

$$
I=2(2 n+1)(m+1)-(m+1)^{2}+1
$$

for the $L[n ; m, k]$ transformation.

The values of the $S[m, k]$ transformations are not included because they, in most cases, lead to poorer results than the other transformations, and $f_{M / N}$ is not included since it is known to converge slower than $[n ; m / k]_{r}[6]$. The considered approximants contain one-dimensional sums, and their accuracy therefore depends both on the order of transformation and of the accuracy by which the one-dimensional sums are calculated. This complicates the comparison, and we have therefore calculated the approximants with very accurate one-dimensional sums. In Table 5.1 the $S^{\prime}[n ; m, k]$ transformation, in most cases, gives the most accurate results, and in Table $5.2 L[n ; m, k]$ is the most accurate.

6. A Conditionally Convergent Series. Numerical Example. Let us consider the double series

$$
\sum_{n=0}^{\infty} \sum_{m=0}^{\infty} \frac{e^{-i k R_{n m}}}{R_{n m}},
$$

where

$$
R_{n m}=\sqrt{x_{n}^{2}+y_{m}^{2}}, \quad x_{n}=n a+d\left(1+(-1)^{n+1}\right), \quad y_{m}=2(m+1) b .
$$

The series originates in calculation of the electrical field in a rectangular waveguide at microwave frequencies.

Attempts to accelerate the convergence of (6.1) have been made using $L[n ; m, k]$, $S^{\prime}[n ; m, k],[n ; m / k]_{r}$ and $S[m, k]$. Reasonable convergence was found for all methods except $L[n ; m, k]$.

The series is conditionally convergent, and the phase of the terms varies in an unsystematical way. Thus the partial sum $A_{s t}$ of (4.9) is a complicated function of $s$ and $t$, and the leading behavior is not as simple as (4.13). This may explain why $L[n ; m, k]$ fails to accelerate the convergence of $(6.1)$. 
Results using $S^{\prime}[n ; m, k]$ and $[n ; m / k]_{r}$ are very similar, and only results of $[n ; m / k]_{r}$ and $S[m, k]$ are presented in the following. Table 6.1 shows a comparison between the $[n ; m / k]_{r}$ and $S[m, k]$ transformation, where the series is calculated with the parameters $a=6, b=2, d=1.5$ and $k=1$. Reference values in the table are obtained by high order Shanks transformation of the one-dimensional sequence,

$$
A_{t}=\sum_{n=0}^{t} \sum_{m=0}^{t} \frac{e^{-i k R_{n m}}}{R_{n m}},
$$

and have been checked using an analytical calculation of (6.1). The slow convergence of the series limits the accuracy of the $[n ; m / k]$, due to the large number of series terms required for the evaluation of the infinite one-dimensional sums. $S[m, k]$ does not involve calculation of one-dimensional sums and gives about the same accuracy with less computational effort.

TABI.E 6.1

Comparison of $[n: m / k]$, and $S[m, k]$ approximants to the series $(6.1)$ for $k=1, a=6, b=2$ and $d=1.5$.

\begin{tabular}{|l|c|c|l|l|} 
& Terms & Equations & Real part & Imag. part \\
\hline Reference value & & & -0.15788505 & 0.12962980 \\
\hline $\mathrm{S}[2,2]$ & 25 & 9 & -0.15989 & 0.13711 \\
$\mathrm{~S}[5,5]$ & 121 & 36 & -0.15755 & 0.12994 \\
$\mathrm{~S}[9,9]$ & 361 & 100 & -0.157882 & 0.129622 \\
{$[9 ; 2 / 2]_{3}$} & 113 & 9 & -0.15618 & 0.12843 \\
{$[9 ; 5 / 5]_{6}$} & 227 & 36 & -0.15787 & 0.129636 \\
{$[9 ; 9 / 9]_{10}$} & 379 & 100 & -0.157883 & 0.129620 \\
\hline
\end{tabular}

7. Conclusion. This paper has presented $M$-dimensional generalizations of Shanks' e-transformation [8] (denoted $S[m, k]$ and $S^{\prime}[n ; m, k]$ ) and of Levin's one-dimensional transformations [7] $(L[n ; m, k])$.

The transformations are compared, numerically, to Levin's 2-dimensional transformation $[n: m, k]$, . From this comparison it is seen that the $S^{\prime}[n ; m, k]$ transformation gives generally higher precision than $[n ; m / k]_{r}$ and $L[n ; m, k]$ for the same number of linear equations solved, but uses more terms of the series. The $L[n ; m, k]$ transformation gives generally better results than $[n ; m / k]_{r}$ and $S^{\prime}[n ; m, k]$ for the same series terms used.

The $S[m, k]$ transformation does not require calculation of infinite one-dimensional series and is therefore efficient when the series is slowly convergent such that these calculations use a large number of series terms. This is illustrated by a numerical example.

Future efforts could be devoted to the invention of recursive algorithms for computation of $\boldsymbol{M}$-dimensional series transformations. For one-dimensional series such algorithms exist for computation of Shanks' $e$-transformation [10] and Levin's transformations [7]. 
8. Acknowledgement. The authors wish to thank the referee for suggesting several improvements of the paper. In particular a change in the presentation of the numerical examples is acknowledged.

Institute of Applied Mathematical Physics

Technical University of Denmark

DK-2800 Lyngby, Denmark

Electromagnetics Institute

Technical University of Denmark

DK-2800 Lyngby, Denmark

Electromagnetics Institute

Technical University of Denmark

DK-2800 Lyngby, Denmark

1. (j. A. Baker, Essentials of Pade Approximants, Academic Press, New York, 1975.

2. C. Brezinski, Accélération de la Concergence en Analyse Numérique, Lecture Notes in Math. Vol. 584. Springer-Verlag, Berlin and New York. 1977.

3. J. S. R. Chishol.M, "Rational approximants defined from double power series," Math. Comp., v. 27. 1973, pp. 841-848.

4. J. S. R. Chishol.M \& J. McEwan, "Rational approximants defined from power series in $N$ variables," Proc. Rol. Soc. (Iondon) Ser. A, v. 336. 1974, pp. 421-452.

5. P. R. Graves - Morris, R. Hughes - Jonis \& (j. J. Makinson, "The calculation of some rational approximants in two variables," J. Inst. Math. Appl., v. 13, 1974, pp. 311-320.

6. D. Levin, "On accelerating the convergence of infinite double series and integrals," Math. Comp. v. $35,1980$. pp. $1331-1345$

7. D. LEVIN, "Development of non-linear transformations for improving convergence of sequences," Internat. J. Comput. Math., v. B3, 1973, pp. 371-388.

8. D. ShanKs, "Non-linear transformations of divergent and slowly convergent sequences," J. Math. Phis., v. 34, 1955, pp. 1-42.

9. R. F. Streit, "The evaluation of double series," BIT, v. 12, 1972, pp. 400-408.

10. P. WYNN, "On a device for computing the $e_{m}\left(S_{n}\right)$ transformation," MTAC, v. 10, 1956, pp. 91-96. 